\title{
Preparation of Controlled-Release Fine Particles Using a Dry Coating Method
}

\author{
Shohei Nakamura, ${ }^{1,3}$ Takatoshi Sakamoto, ${ }^{1}$ Tomonori Ito, ${ }^{1}$ Kazuhiro Kabasawa, ${ }^{2}$ and Hiroshi Yuasa ${ }^{1}$
}

Received 28 September 2015; accepted 21 December 2015; published online 14 January 2016

\begin{abstract}
Wet coating methods use organic solvents to prepare layered particles that provide controlledrelease medications. However, this approach has disadvantages in that it can cause particle agglomeration, reduce pharmaceutical stability, and leave residual organic solvents. We used a dry coating method to overcome these issues. Fine particles (less than $50 \mu \mathrm{m}$ in diameter) of controlled-release theophylline were created using theophylline (TP; model drug), polyethylene glycol 20,000 (PEG; drug fixative), hydrogenated castor oil (HCO; controlled-release material), hydrogenated rapeseed oil (HRSO; controlledrelease material), and cornstarch (CS; core particle). An ultrahigh-speed mixer was employed to mix $\mathrm{TP}$ and CS for $5 \mathrm{~min}$ at 28,000 rpm. Subsequent addition of PEG produced single-core particles with a drug reservoir coating. Addition of HCO and HRSO to these particles produced a controlled-release layer on their surface, resulting in less than $10 \%$ TP dissolution after $8 \mathrm{~h}$. We successfully demonstrated that this dry coating method could be used to coat $16-\mu \mathrm{m}$ CS particles with a drug reservoir layer and a controlledrelease layer, producing multi-layer coated single-core particles that were less than $50 \mu \mathrm{m}$ in diameter. These can be used to prepare controlled-release tablets, capsules, and orally disintegrating tablets.
\end{abstract}

KEYWORDS: controlled-release fine particles; drug reservoir coating; dry coating; multi-layer coating; theophylline.

\section{INTRODUCTION}

Recent developments in oral drug formulation include attempts to generate controlled-release forms of various target drugs. Compared to pharmaceuticals with rapid-release properties, controlled-release drugs can be prescribed less frequently, have prolonged efficacy, possess fewer side effects and toxicity, and enhance a patient's quality of life.

There are two types of controlled-release formulations: multiple-unit and single-unit. For physiological reasons, multipleunit controlled-release formulations exhibit lower dose dumping and less variability between lots, as well as a higher level of safety, as compared to single-unit controlled-release formulations $(1,2)$.

To prepare multiple-unit formulations, drug particles are generally granulated in advance. The widely used wet coating method involves coating drug granules with controlled-release materials dissolved in an organic solvent or mixed as a dispersion. However, forming a controlled-release layer in this manner requires a significant amount of time and a large amount of organic solvent, which may lead to numerous problems such as end-product residues, damage to manufacturing workers' health, and detrimental effects on the environment.

\footnotetext{
${ }^{1}$ Department of Pharmaceutical Technology, College of Pharmaceutical Sciences, Matsuyama University, 4-2 Bunkyo-cho, Matsuyama, Ehime 790-8578, Japan.

${ }^{2}$ Tokyo Printing Ink Manufacturing Company Limited, 1-397 Yoshino-cho, Kita-ku, Saitama, Saitama 331-0811, Japan.

${ }^{3}$ To whom correspondence should be addressed. (e-mail: snakamur@cc.matsuyama-u.ac.jp)
}

In recent years, a dry coating method that does not involve organic solvents has gained attention. This avoids the issues stemming from the use of organic solvents and is believed to be suitable for heat-labile drugs. Shimono et al. reported the preparation of a drug-containing pellet using a granulation apparatus, hydrogenated castor oil, and magnesium stearate. This product possessed controlled-release properties owing to its multi-layer coating (3). Additionally, Pearnchob and Bodmeier prepared a granule with controlled-release properties using a fine powder coating of methacrylate copolymers and a Wurster fluidized bed coating apparatus (4). The disadvantages of these two approaches include the need for extended periods of heat treatment and the use of liquid plasticizers. In contrast, Fujimoto et al. coated ethylcellulose core particles (jet mill-pulverized) with crystallized cellulose or crystallized lactose using a twin-screw continuous kneader (5), and Kondo et al. prepared controlled-release composite particles by processing a poly(meth)acrylate controlledrelease material (finely powdered by rapid spraying and freeze drying) and a mechanically rounded drug using a mechanical powder processor (6). However, these methods require that all coating materials first be micronized or powdered.

In previous studies, we reported the preparation of controlled-release particles by combining nonmicronized drugs, coating layer powders, and core particles (approximately $250 \mu \mathrm{m}$ in diameter) in a mixing apparatus and processing them by ultrahigh-speed mixing, resulting in the formation of a drugcontaining matrix layer on the surface of the core particles within several minutes via dry coating (7). However, there was a concern that this process led to significant variability in tablet weights and 
Table I. Physical Properties of the Raw Materials

\begin{tabular}{llllll}
\hline Material & Particle size $(\mu \mathrm{m})$ & True density $(\mathrm{g} / \mathrm{mL})$ & Tap density $(\mathrm{g} / \mathrm{mL})$ & Melting point $\left({ }^{\circ} \mathrm{C}\right)$ & Viscosity $(\mathrm{mPa})$ \\
\hline TP & 63.2 & 1.47 & 0.64 & 273 & - \\
CS & 15.9 & 1.51 & 0.68 & - & - \\
PEG & 441.4 & 1.24 & 0.57 & 61.8 & $41,048.1$ \\
HCO & 26.2 & 1.02 & 0.48 & 85.5 & 40.9 \\
HRSO & 13.6 & 1.01 & 0.29 & 68.4 & 20.0 \\
\hline
\end{tabular}

TP theophylline, $C S$ cornstarch, $P E G$ polyethylene glycol 20,000, HCO hydrogenated castor oil, $H R S O$ hydrogenated rapeseed oil

drug content, and the particles might be destroyed during compression to tablet form, owing to the large diameter of the particles. In addition, the mean particle size in orally disintegrating controlled-release tablets should not exceed $50 \mu \mathrm{m}$, in order to prevent discomfort caused by rough texture (8-13). To address these issues, we used a dry coating method to prepare controlledrelease fine particles with diameters of less than $50 \mu \mathrm{m}$ (difficult to achieve with wet coating (14)) containing two layers: a drug reservoir layer and a controlled-release layer. These particles are ideal for use in controlled-release tablets and capsules, and they can also be used to create controlled-release orally disintegrating tablets.

\section{MATERIALS AND METHODS}

\section{Materials}

Anhydrous theophylline (TP; Wako Pure Chemicals Industries, Osaka, Japan) was used as the model drug, cornstarch (CS; Nihon Shokuhin Kako Corp., Tokyo, Japan) was used as the core particle, polyethylene glycol 20,000 (PEG; NOF Corp., Tokyo, Japan) was used to fix the drug, hydrogenated castor oil (HCO; Lubliwax 101, Freund Corp., Tokyo, Japan) and hydrogenated rapeseed oil (HRSO; Lubliwax 103, Freund Corp., Tokyo, Japan) were used as controlled-release agents, and light anhydrous silicic acid (LAS; Aerosil R972, Nippon Aerosil, Tokyo, Japan) was used to prevent granulation.

Table II. Quantities Used During Dry Coating

\begin{tabular}{lr}
\hline CS-R preparation \\
CS & 7.28 \\
TP & 1.44 \\
PEG & 2.40
\end{tabular}

HCO coating

\begin{tabular}{llll} 
& \multicolumn{3}{l}{ HCO layer thickness } \\
& $(0.5 \mu \mathrm{m})$ & $(1.0 \mu \mathrm{m})$ & $(1.5 \mu \mathrm{m})$ \\
CS-R & 9.56 & 8.31 & 7.26 \\
HCO & 1.12 & 2.04 & 2.82
\end{tabular}

HRSO coating

\begin{tabular}{lllll} 
& \multicolumn{4}{l}{ HRSO layer thickness } \\
& $(3.0 \mu \mathrm{m})$ & $(5.0 \mu \mathrm{m})$ & $(7.0 \mu \mathrm{m})$ & $(9.0 \mu \mathrm{m})$ \\
CS-R/HCO & 3.62 & 2.25 & 1.51 & 1.07 \\
HRSO & 3.17 & 3.85 & 4.21 & 4.43 \\
\hline
\end{tabular}

All values represent $\mathrm{g}$

$C S$ cornstarch, $C S-R$ CS particles coated with a drug reservoir layer, $H C O$ hydrogenated castor oil, HRSO hydrogenated rapeseed oil, $P E G$ polyethylene glycol 20,000, TP theophylline

\section{Particle Size Distribution and Average Particle Diameter Measurements}

The particle size distribution for each sample powder was measured using a laser diffraction particle size analyzer (SALD-2200; Shimadzu Corp., Kyoto, Japan). The particle size distribution was measured using a cyclone injection-type dry measurement unit (SALD-DS5; Shimadzu Corp., Kyoto, Japan) with a stage elevation rate of $10 \mathrm{~mm} / \mathrm{s}$, a compressed air pressure of $0.5 \mathrm{MPa}$, and a refractive index of $1.60-0.10 i$. From the particle size distribution, we determined the median diameter; this was used when deciding on a coating prescription amount (based on coating layer thickness).

\section{Micronization of HRSO}

As purchased, the average particle diameter of HRSO is approximately $100 \mu \mathrm{m}$, which is large compared to the CS core particles. We therefore micronized HRSO by first suspending $15 \mathrm{~g}$ of HRSO in $100 \mathrm{~mL}$ of ethanol at approximately $70^{\circ} \mathrm{C}$ and stirring until the solution formed a transparent emulsion. After terminating heating and agitation, the cloudy suspension (caused by separation of the HRSO) was processed for 30 min using an homogenizer (T10 basic Ultra-Turrax; IKAWerke GmbH \& Co. KG, Staufen, Germany). The suspension was transferred to a centrifuge tube and centrifuged at $3000 \mathrm{rpm}$ for $10 \mathrm{~min}$ at $5^{\circ} \mathrm{C}$ (CF16RX II; Hitachi Koki Co., Ltd., Tokyo, Japan). The clear supernatant was removed and the sediment was vacuum-dried and lightly ground in an agate mortar to produce the micronized HRSO used for dry coating.

\section{Measurement of True and Tap Densities}

Using a helium gas automatic pycnometer (Ultrapyc1200e; Quantachrome, Boynton Beach, FL, USA), the true density of approximately $10 \mathrm{~g}$ of each powder was measured. In order to determine the tap density, approximately $50 \mathrm{~g}$ of each powdered material was gently placed in a dry 100-mL graduated cylinder without consolidation. The volume was measured after dropping the cylinder 50 times from a height of $5 \mathrm{~cm}$ to settle the powder naturally. The tap density of each powder was calculated from the measured volume and weight.

\section{Measurement of Viscosity}

The melting viscosities of the coating agents (PEG, HCO, and HRSO) were measured using a cone-plate rotational viscometer (RVDV-III Ultra; Brookfield, Middleboro, MA, USA). A 1-mL sample of each melted coating material was 


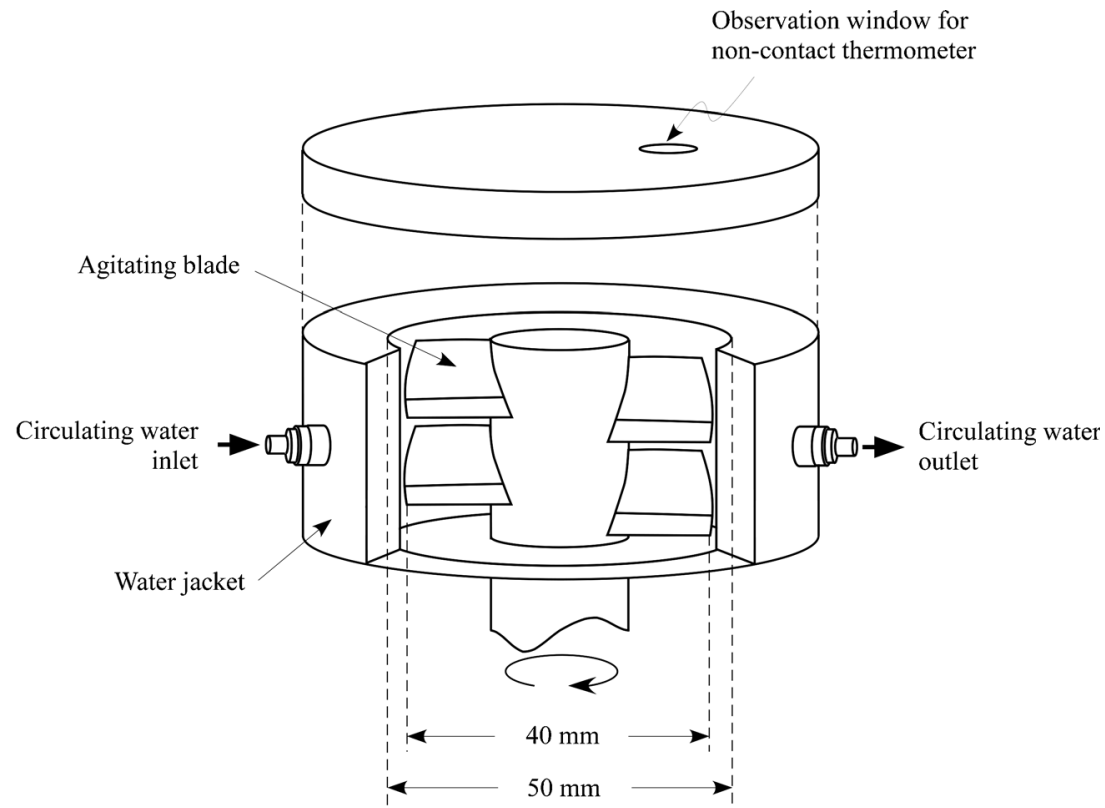

Fig. 1. Schematic of the ultrahigh-speed mixer used for dry coating. The agitating blade is rotatable at $28,000 \mathrm{rpm}$ and the temperature of the apparatus is controlled by circulating water during agitation

placed in a measuring container, and the viscosity at five different temperatures (above the melting point) was measured. The viscosity at each respective melting point was calculated according to the Andrade equation (15).

\section{Measurement of Melting Point and Enthalpy of Fusion}

The melting point and enthalpy of fusion for each powder was measured by differential scanning calorimetry (DSC; Thermo Plus DSC8230; Rigaku, Tokyo, Japan). Approximately $10 \mathrm{mg}$ of each powder was accurately weighed and sealed in an aluminum sample pan. The melting point and enthalpy of fusion were determined from the DSC curve obtained under a temperature range of $30-120^{\circ} \mathrm{C}$, with a rate of temperature increase of $5^{\circ} \mathrm{C} / \mathrm{min}$ and increasing internal atmospheric temperature.

\section{Coating Prescription}

The coating prescription for each powder was calculated based on its true density, tap density, and average particle diameter (Table I). For the required amount of coating, suitable layer thicknesses were calculated based on the assumption that each added layer that formed on the CS particle would not contain any empty space. For the drug reservoir layer, $0.5 \mu \mathrm{m} \mathrm{TP}$ and $1.0 \mu \mathrm{m}$ PEG were coated
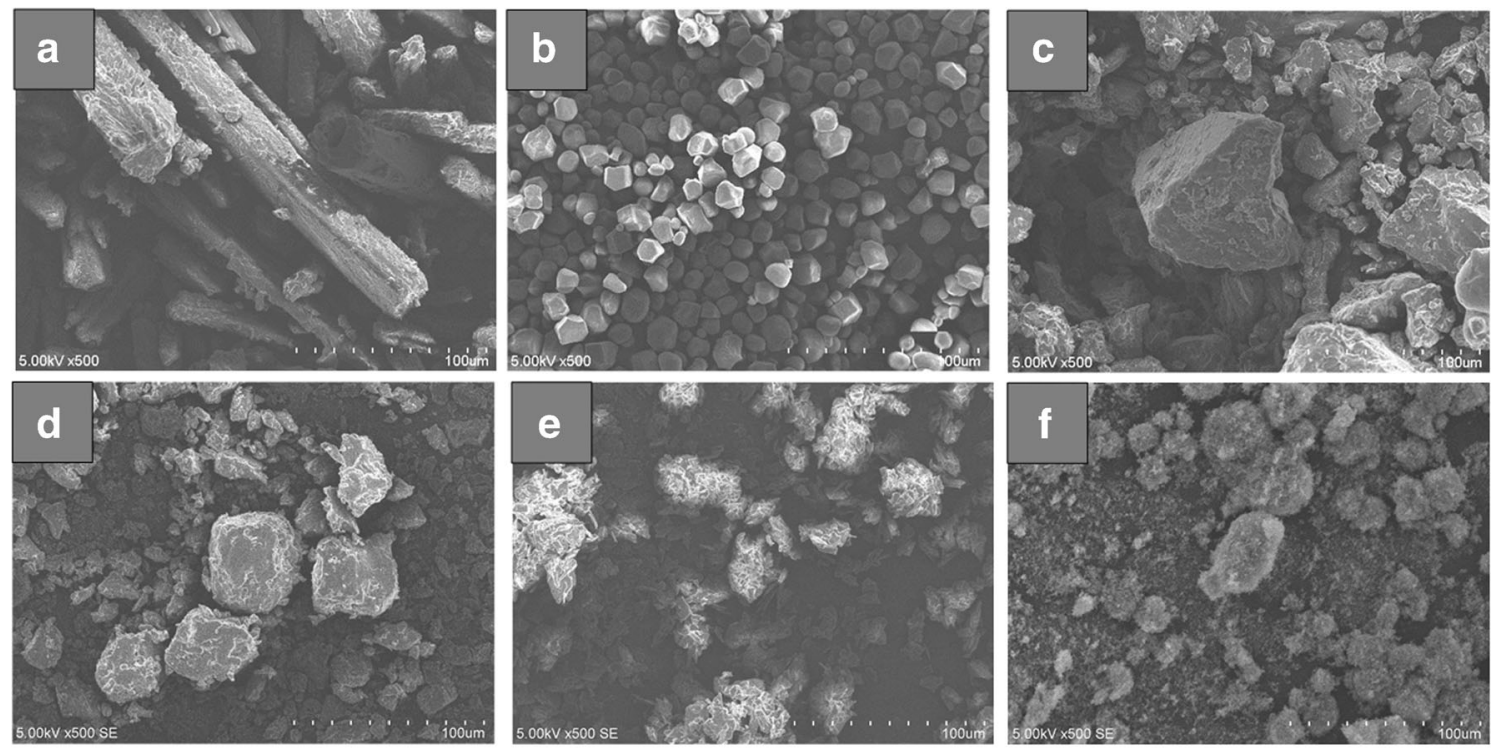

$100 \mu \mathrm{m}$

Fig. 2. SEM images of the raw materials. a TP, b CS, c PEG, $\mathbf{d}$ HCO, e micronized HRSO, and $\mathbf{f}$ LAS. Scale bar $=100 \mu \mathrm{m}$ 

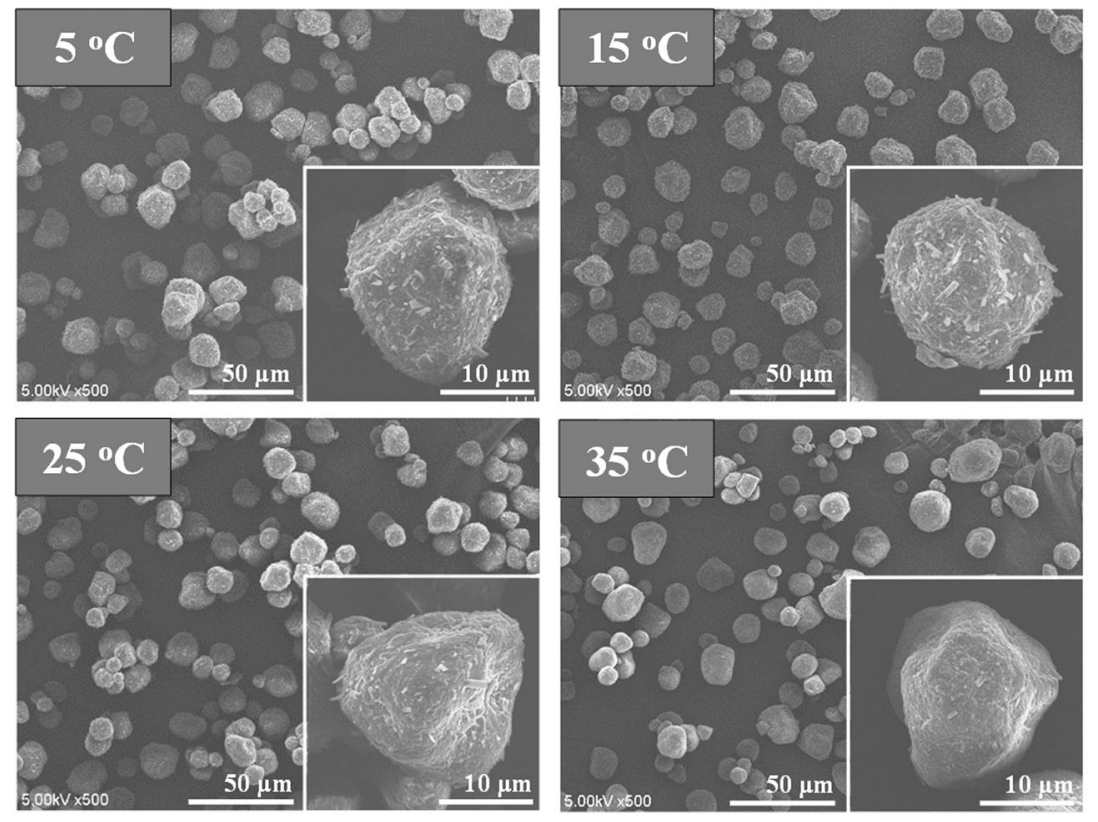
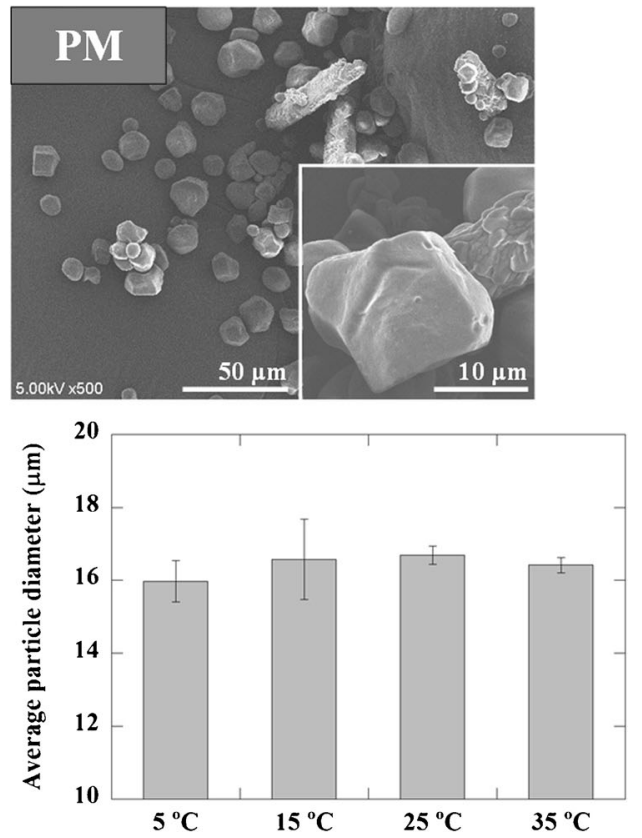

Fig. 3. SEM images of the drug reservoir layer on the CS particles at the indicated preparation temperatures, and of the physical mixture (PM) of CS, TP, and PEG. The magnified image of a single particle is shown in the lower right-hand corner of each low-magnification image. The scale bars are indicated on each image. The lower right-hand panel shows the average coated particle diameter at the indicated temperature $(n=3)$

onto the CS particle. For the controlled-release layer, 0.5$1.5 \mu \mathrm{m} \mathrm{HCO}$ and 3-9 $\mu \mathrm{m}$ HRSO formed a laminating layer on the drug reservoir layer. To prevent agglomeration and granulation, $1.0 \%$ LAS was added before coating with each controlled-release layer. The actual prescription amounts are listed in Table II.
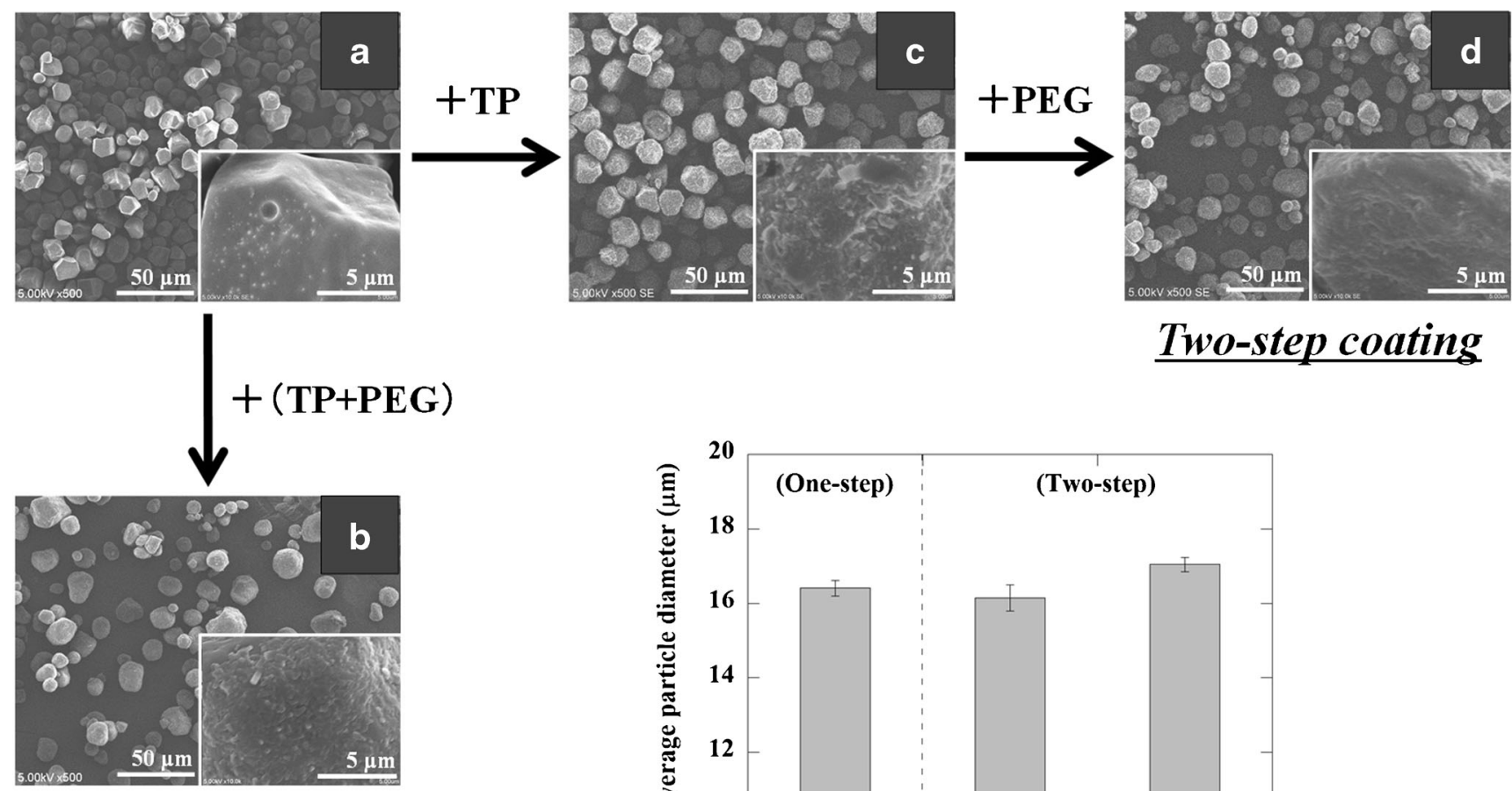

\section{One-step coating}

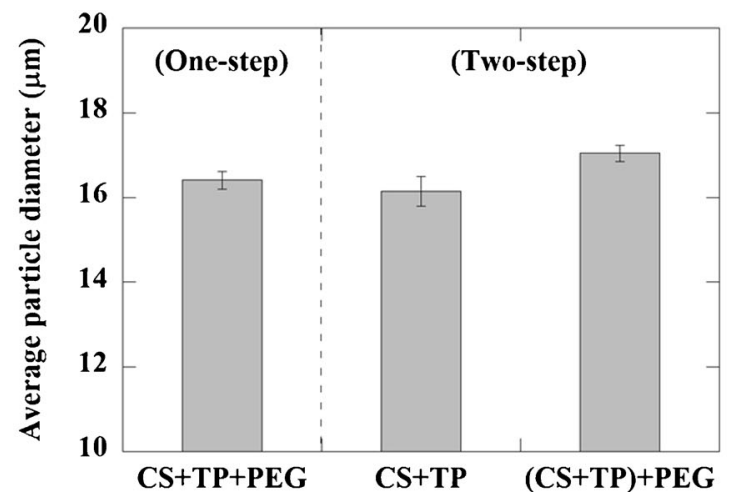

Fig. 4. SEM images of particles during one-step $(\mathbf{a}, \mathbf{b})$ or two-step $(\mathbf{a}, \mathbf{c}-\mathbf{d})$ coating at $35^{\circ} \mathrm{C}$. a CS, $\mathbf{b}$ one-step CS particle coating with TP and PEG, $\mathbf{c}$ CS particle coated with a TP layer, $\mathbf{d}$ CS particle coated with a TP layer, followed by a PEG layer. A magnified image of the particle surface is shown in the lower right-hand corner of each low-magnification image. The scale bars are indicated on each image. The lower righthand panel shows the average coated particle diameter $(n=3)$ 


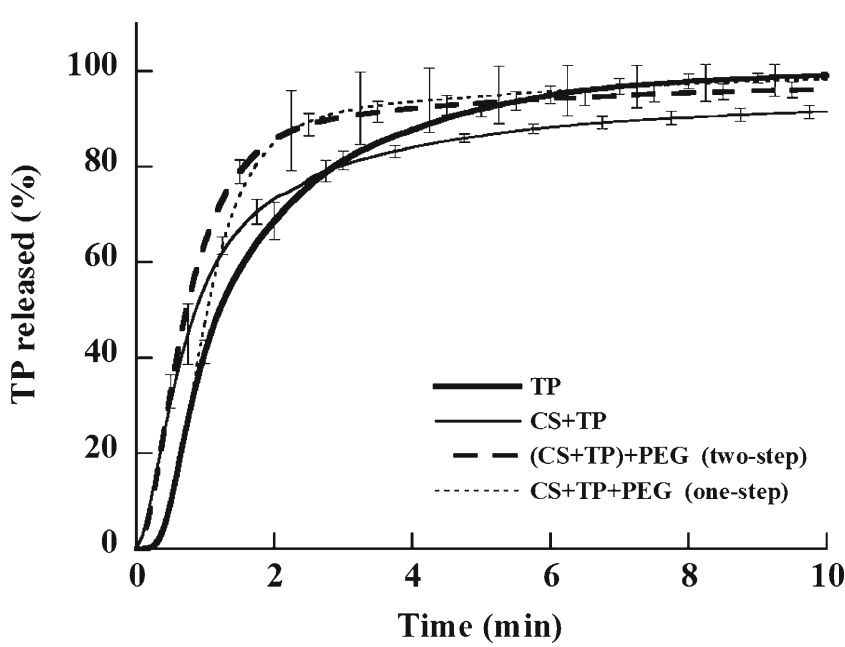

Fig. 5. TP dissolution behavior from raw TP powder, one-step coated particles, TP-layered particle, and two-step coated particles in water. Data represent the mean $\pm \operatorname{SD}(n=3)$

\section{Dry Coating}

Dry coating was carried out using an ultrahigh-speed mixer (Fascinate ${ }^{\circledR}$; Tokyo Printing Ink Mfg. Co., Ltd., Tokyo, Japan), as shown in Fig. 1. According to the determined prescription, the appropriate amount of powder was added to the mixing vessel (filled to $30 \%$ capacity) and mixed at a speed of 28,000 rpm (outer agitation blade tip speed: approximately $65 \mathrm{~m} / \mathrm{sec}$ ) for $5 \mathrm{~min}$. During mixing, the mixing vessel temperature was regulated by a fluid convection system. The drug reservoir layer was formed at $35^{\circ} \mathrm{C}$, while the controlledrelease layer formed at $5^{\circ} \mathrm{C}$.

\section{Evaluation of TP Dissolution}

The dissolution rate of TP from the coated particles was evaluated by a dissolution test, in accordance with the paddle method prescribed in the sixteenth edition of the Japanese Pharmacopoeia. Using a coated particle containing $10 \mathrm{mg}$ of TP and a flow cell system (dissolution test apparatus: NTR-

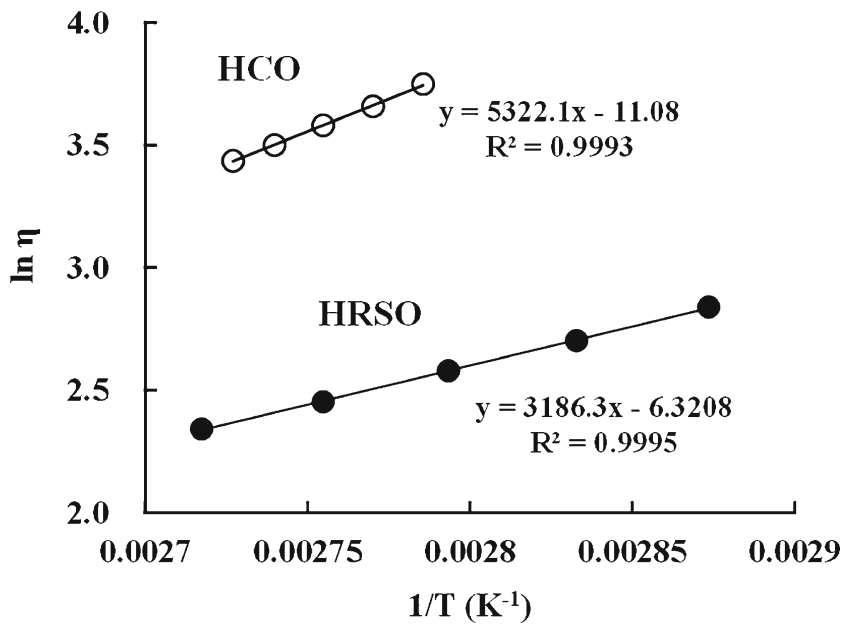

Fig. 6. Relationship between the log of viscosity $(\eta)$ and the inverse temperature $(\mathrm{K})$ for $\mathrm{HCO}$ and HRSO. The equations of the regression line are indicated, with $R^{2}$ (coefficient of determination)
1000; Toyama Sangyo Co., Osaka, Japan; UV-absorption photometer: U-1900; Hitachi High-Technologies Corp., Tokyo, Japan), the absorbance was measured at a wavelength of $271 \mathrm{~nm}$ to determine the dissolution of TP over time. Distilled water was used as the test fluid, and measurements were taken at a paddle rotation speed of $50 \mathrm{rpm}$. For determination of the TP dissolution rate, the amount of TP in the test fluid was converted to a percentage, based on the prescribed TP content (set at $100 \%$ ).

\section{Analysis of Particle Shape and Surface Conditions}

The raw powders and coating particles were fixed to the sample stage by conductive carbon tape, and an ion sputtering apparatus (E-1010; Hitachi High-Technologies Corp.) was used for platinum deposition, which was carried out under low pressure for $10 \mathrm{~s}$. The particle shape and surface conditions were observed by scanning electron microscopy (SEM; S-3400N; Hitachi High-Technologies Corp.) under an acceleration voltage of $5 \mathrm{kV}$.

\section{TP Crystallinity}

Using a powder X-ray diffractometer (RINT-Ultima III; Rigaku, Tokyo, Japan), the crystallinity of raw TP powder and coated particles was evaluated based on diffraction intensities obtained using a diffraction angle of $2 \theta=5-40^{\circ}$, a $\mathrm{Cu}$ target, a tube voltage of $40 \mathrm{kV}$, and a tube current of $40 \mathrm{~mA}$.

\section{RESULTS AND DISCUSSION}

\section{Formation of the Drug Reservoir Layer}

We examined the effect of vessel temperature on the formation of the drug reservoir layer on CS particles. Figure 2 shows SEM images of the raw materials, and Fig. 3 shows the physical mixture (PM), as well as the shape and surface condition of the particles obtained after addition of TP and PEG to $\mathrm{CS}$ over a temperature range of $5-35^{\circ} \mathrm{C}$. The $\mathrm{PM}$ retained the original shape of the individual powder particles. Owing to agitation, PEG affixed the pulverized TP to the surface of the CS particles at all temperatures tested. From 5 to $25^{\circ} \mathrm{C}$, TP microcrystals adhered to the surface of CS particles. At higher temperatures $\left(35^{\circ} \mathrm{C}\right)$, the surface of the particles became smooth. We believe that an outermost smooth drug reservoir layer had formed because melted PEG did not exhibit cracking on the particle surface. The bar chart in Fig. 3 (lower right panel) indicates the average particle diameter of the coated particles prepared at each temperature $(n=3)$. The variation in the average particle diameter was small at each temperature and showed good reproducibility. Granulation occurred when the materials were mixed at $45^{\circ} \mathrm{C}$, making preparation of a single-core particle difficult. We suspect that when the initial temperature of the powder was too high, the added energy and mechanical heat generated by the mixing caused the PEG to completely melt and act as a binding agent. Differences in the TP X-ray diffraction patterns and intensities between the resulting single-core particles and the physical mixture of TP crystals were barely noticeable. Therefore, the mixing process seemed to cause little, if any, degradation of TP crystals. 

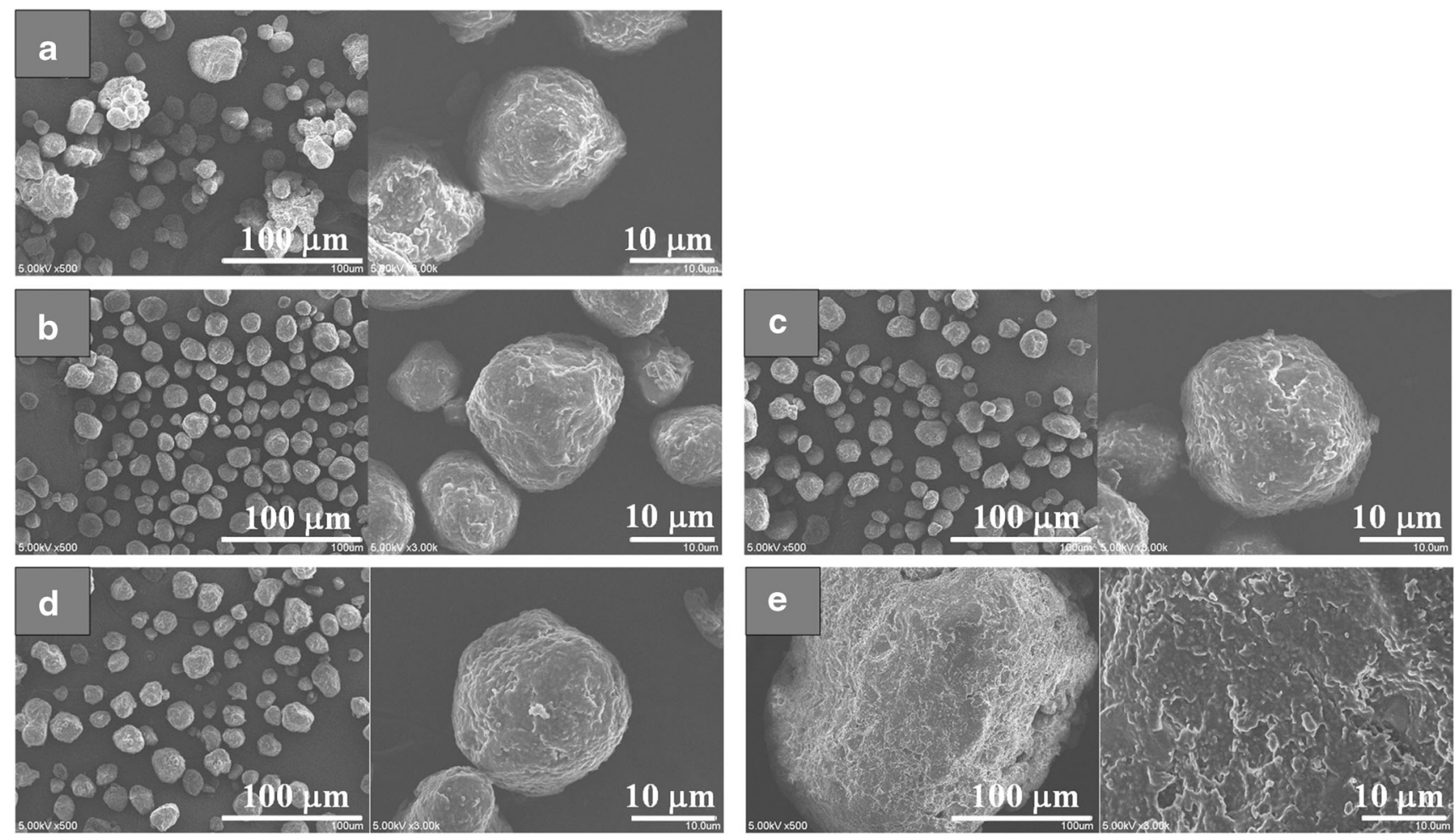

Fig. 7. Effects of vessel temperature and $\mathrm{HCO}$ layer thickness on the coating. a $0.5 \mu \mathrm{m} \mathrm{HCO}\left(35^{\circ} \mathrm{C}\right), \mathbf{b} 0.5 \mu \mathrm{m} \mathrm{HCO}\left(5^{\circ} \mathrm{C}\right)$, $\mathbf{c} 1.0 \mu \mathrm{m} \mathrm{HCO}\left(5^{\circ} \mathrm{C}\right)$, d: $1.5 \mu \mathrm{m} \mathrm{HCO}\left(5^{\circ} \mathrm{C}\right), \mathbf{e} 2.0 \mu \mathrm{m} \mathrm{HCO}\left(5^{\circ} \mathrm{C}\right)$. The left-hand panel in each image shows a low-magnification image and the right-hand panel shows a high-magnification image of a single particle. The scale bars are indicated on each image

Next, we examined the effects of different mixing procedures. Figure 4 shows SEM images of particle surfaces after CS, TP, and PEG were mixed together simultaneously or in stages at $35^{\circ} \mathrm{C}$. As shown in Fig. $4 \mathrm{a}$, the surface of CS particles (which will become core particles) was smooth, with little unevenness. When CS, TP, and PEG were added simultaneously, micronized TP was fixed by PEG, but the TP on the particle surface was not completely covered by PEG, giving it a rough surface (Fig. 4b). This was likely caused by simultaneous spreading of the pulverized TP and melting of PEG, resulting in

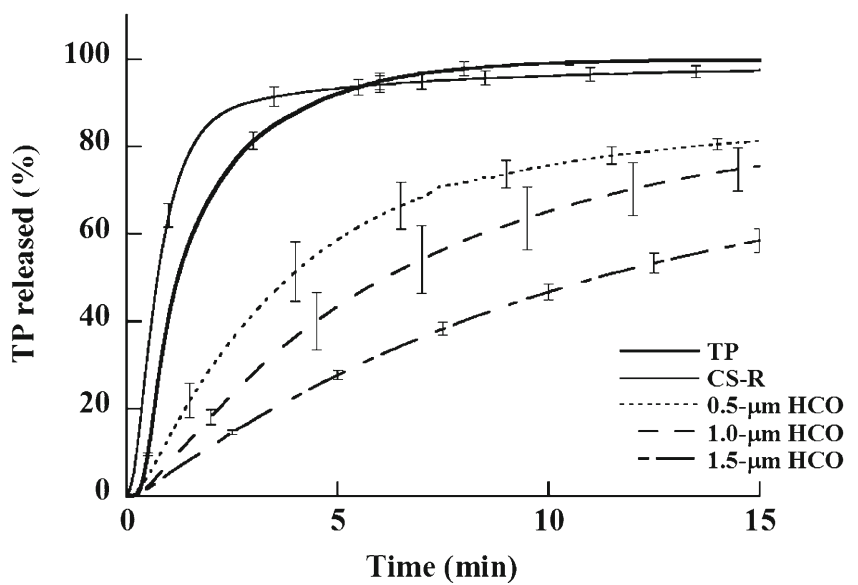

Fig. 8. TP dissolution behavior from TP powder, CS-R particles, and particles with HCO layers at the indicated thickness in water. Data represent the mean $\pm \mathrm{SD}(n=3)$ failure of the melted PEG to coat all of the dispersed TP particles. Accordingly, mixing was then divided into a twostep procedure whereby CS and TP were mixed in advance, and PEG was added subsequently. Figure 4c shows the surface condition of the particles that resulted from mixing CS and TP prior to adding PEG. Theophylline was pulverized when mixed with CS, and thus stuck to the surface of the CS particle, forming an embedded TP layer. Additionally, TP particles that adhered to the surface of the CS particles were smaller in size than those produced during the one-step procedure. We surmise that in the two-step process, the CS particles assist in pulverizing TP particles to submicron size, because the CS particles are smaller and harder than TP particles and PEG is not present to act as a shock-absorber. Figure $4 d$ shows the surface condition of the particles obtained following the second mixing step, when PEG was added to the TPcoated CS particles. PEG coated the TP-covered CS particles, resulting in an extremely smooth surface. The particles obtained by mixing with PEG were all single-core particles, and we were able to form a drug reservoir layer composed of TP and PEG on the surface of each CS particle. The bar chart in Fig. 4 (lower right panel) indicates the average particle diameter of the coated particles prepared by one- and two-step coating $(n=3)$. The variation in the average particle diameter was small in each case and showed good reproducibility. Hereafter, CS particles coated with a drug reservoir layer comprised of TP and PEG will be abbreviated as "CS-Rs" or referred to as the drug reservoir particles. 

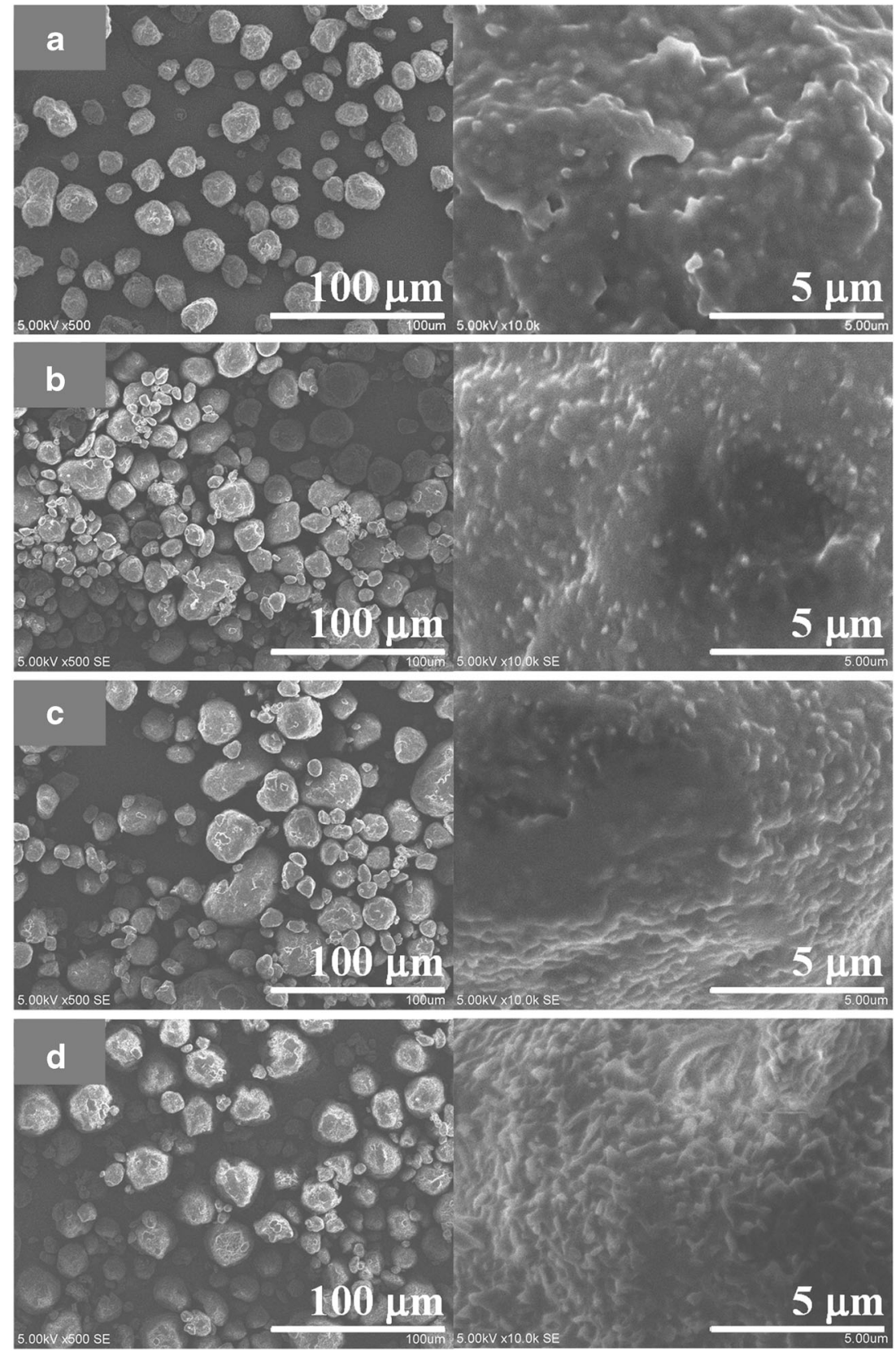

Fig. 9. SEM images of particles coated in $1.5-\mu \mathrm{m} \mathrm{HCO}$, as well as particles coated with the indicated thicknesses of HRSO on the HCO layer. a $1.5 \mu \mathrm{m} \mathrm{HCO}, \mathbf{b} 3.0 \mu \mathrm{m}$ HRSO, c $5.0 \mu \mathrm{m}$ HRSO, d $7.0 \mu \mathrm{m}$ HRSO. The left-hand panel in each image shows a low-magnification image and the right-hand panel shows a high-magnification image of a single particle. The scale bars are indicated on each image

Figure 5 shows the dissolution behavior of TP from raw TP powder, TP-coated CS particles, and CS-Rs derived from the one-step or two-step process. For the TP powder, a sudden increase in dissolution rate was observed early in the experiment, with $80 \%$ dissolution of TP after approximately 3 min, and $100 \%$ dissolution of TP after approximately $10 \mathrm{~min}$. In comparison, the dissolution rate of TP from particles formed via the one- and two-step procedures was almost the same as the dissolution rate of TP from raw powder, with $80 \%$ dissolution of TP within $2 \mathrm{~min}$. It is thought that the pulverized TP, which was distributed throughout the water-soluble PEG layer, was easily saturated by water.

\section{Viscosity of the Materials for Formation of the Controlled- Release Layer}

In order to clarify the mechanism of controlled-release layer formation, an analysis of the viscosities of HCO and HRSO in the melted state was carried out. The viscosities of $\mathrm{HCO}$ and $\mathrm{HRSO}$ were measured at $5^{\circ} \mathrm{C}$ above their melting points, followed by four successive measurements at temperature increments of $5^{\circ} \mathrm{C}$. Although measurements were taken at different shear rates, changing the shear rate did not alter the measured viscosity. Figure 6 shows the relationship between the absolute temperature and viscosity. In 


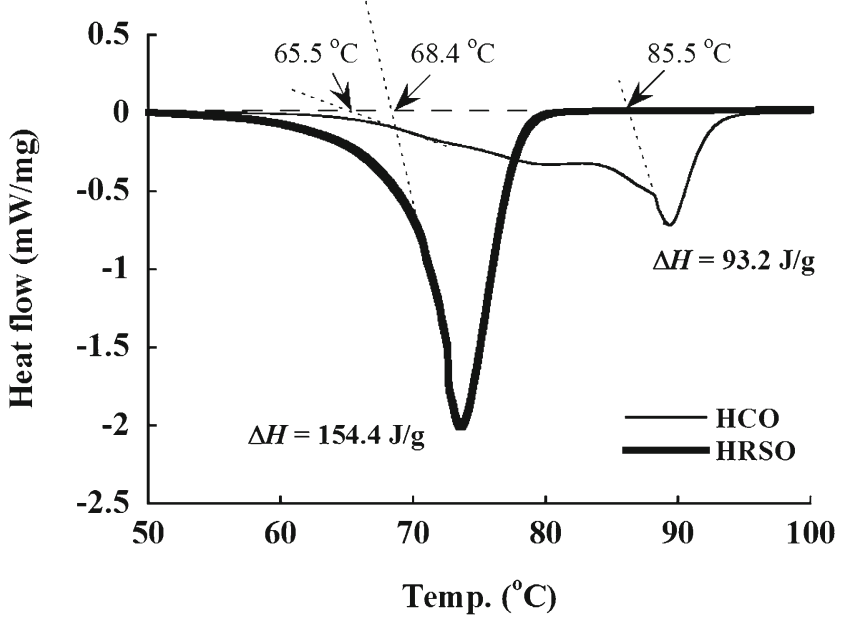

Fig. 10. Melting points, enthalpies of fusion, and DSC curves for $\mathrm{HCO}$ and HRSO. Each melting point was determined from the intersection of the baseline and the tangent line of the DSC curve

accordance with the Andrade equation, a linear relationship was observed between the inverse absolute temperature and the log of viscosity for both HCO and HRSO. The activation energy of flow, determined from the slope of the resultant linear regression approximation equation, was $44.2 \mathrm{~kJ} /$ mol for $\mathrm{HCO}$ and $26.5 \mathrm{~kJ} / \mathrm{mol}$ for HRSO. Compared to HRSO, a larger amount of energy was required to cause melted HCO to flow, signifying highly temperaturedependent viscosity.

During dry coating, particle collisions, shearing (from the agitation blade), and centrifugal force generated by mixing take place. To keep the melted coating material adhered to the surface of the CS-Rs, we attempted to form a controlledrelease layer on the CS-Rs' surface using HCO, a highly viscous hydrophobic material with a higher melting point than HRSO.
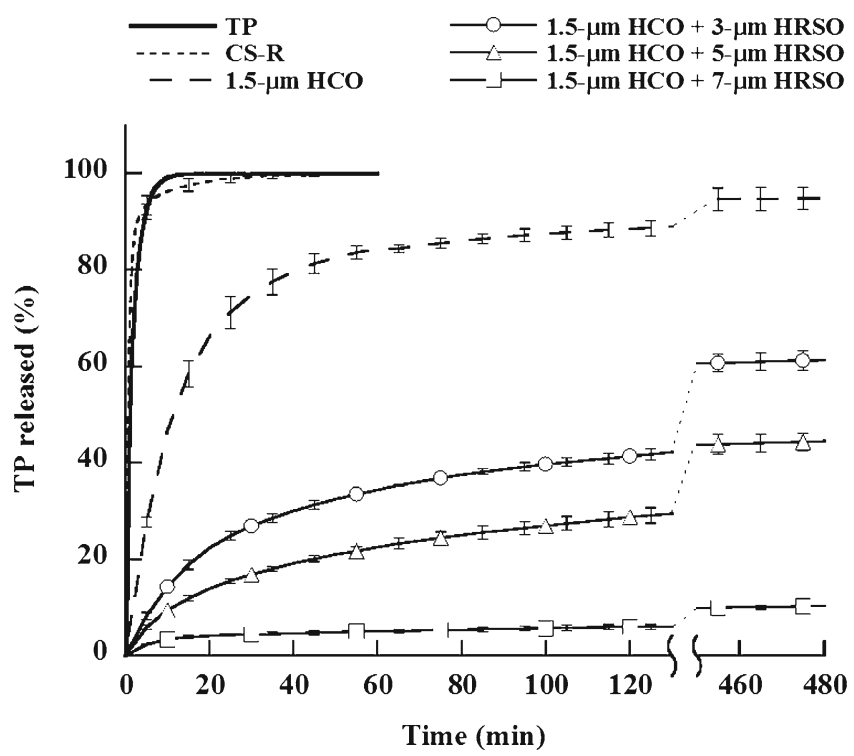

Fig. 11. TP dissolution behavior from TP powder, CS-R, and particles coated in $1.5 \mu \mathrm{m}$ of $\mathrm{HCO}$ and the indicated thicknesses of HRSO in water. Data represent the mean $\pm \mathrm{SD}(n=3)$
Figure 7 shows how the dry coating temperature and the amount of HCO added affected the size and surface conditions of the prepared particles. First, we examined the effect of temperature during mixing. Mixing was carried out by adding CS-Rs to $\mathrm{HCO}$ (in quantities calculated to create a $0.5-\mu \mathrm{m}$ thick layer on the CS-Rs' surface) at the indicated temperatures. At a vessel temperature of $35^{\circ} \mathrm{C}$ (Fig. 7a), granulation caused by melted $\mathrm{HCO}$ prevented the formation of single-core particles. At $5^{\circ} \mathrm{C}$ (Fig. 7b), granulation did not occur and we observed $\mathrm{HCO}$ coating the CS-R surface. In this way, we demonstrated that single-core particles can be formed by coating CS-Rs in $\mathrm{HCO}$, but cooling the vessel to $5^{\circ} \mathrm{C}$ is required for mixing.

Next, the thickness of the HCO layer was varied to investigate how this affected the properties of the particle surface. We mixed $\mathrm{HCO}$ with $\mathrm{CS}-\mathrm{Rs}$ at $5^{\circ} \mathrm{C}$ to prepare 1.0$\mu \mathrm{m}$ (Fig. 7c) or 1.5- $\mu \mathrm{m}$ (Fig. 7d) controlled-release layers on single-core particles. Granulation occurred when a $2.0-\mu \mathrm{m}$ layer of HCO was added (Fig. 7e). This demonstrated that melted HCO on the surface of CS-Rs may inhibit granulation (rather than cause CS-Rs to adhere to each other) by rapidly solidifying at $5^{\circ} \mathrm{C}$. However, we believe that excess melted HCO acted as a binding agent, forming liquid bridges between particles that hardened when rapidly cooled. The inter-particle bonds were strengthened by this process, making preparation of single-core particles difficult (16). These results showed that CS-Rs coated with a controlledrelease layer of up to $1.5 \mu \mathrm{m}$ of $\mathrm{HCO}$ could be manufactured by mixing the ingredients at a vessel temperature of $5^{\circ} \mathrm{C}$.

Figure 8 shows TP dissolution from particles coated with the indicated thickness of HCO. Dissolution of TP from $0.5-\mu \mathrm{m}$ HCO-coated particles was slightly delayed, as compared with dissolution from raw TP powder. This indicates that permeability of the drug reservoir layer to the test fluid was constrained by the hydrophobicity of the HCO-coated CS-R. Furthermore, the TP dissolution rate decreased as the thickness of the HCO layer increased. However, even with a $1.5-\mu \mathrm{m}$ HCO layer (the maximum thickness achievable during the preparation of single-core particles), we were unable to completely inhibit TP dissolution. We believe that particle collisions and shearing cause melted HCO to form a layer on each CS-R. However, cracks in the HCO layer occur when HCO accumulates and hardens without sufficient spreading. Sealing those cracks was necessary to further inhibit TP dissolution.

Table III. The Effects of Coating Thickness on TP Release Parameters

\begin{tabular}{llll}
\hline Coating layer & $K \times 10^{2}$ & $\mathrm{n}$ & $R^{2}$ \\
\hline HCO $0.5 \mu \mathrm{m}$ & 12.70 & 0.991 & 0.984 \\
HCO $1.0 \mu \mathrm{m}$ & 10.45 & 0.781 & 0.992 \\
HCO $1.5 \mu \mathrm{m}$ & 7.41 & 0.770 & 0.984 \\
HCO $1.5 \mu \mathrm{m}+$ HRSO $3 \mu \mathrm{m}$ & 4.53 & 0.478 & 0.860 \\
HCO $1.5 \mu \mathrm{m}+$ HRSO $5 \mu \mathrm{m}$ & 3.04 & 0.472 & 0.905 \\
HCO $1.5 \mu \mathrm{m}+$ HRSO $7 \mu \mathrm{m}$ & 1.25 & 0.351 & 0.783 \\
\hline
\end{tabular}

$H C O$, hydrogenated castor oil, $H R S O$ hydrogenated rapeseed oil, $K$ kinetic constant $\left(\min ^{-n}\right), n$ diffusional exponents, $R^{2}$ coefficient of determination 


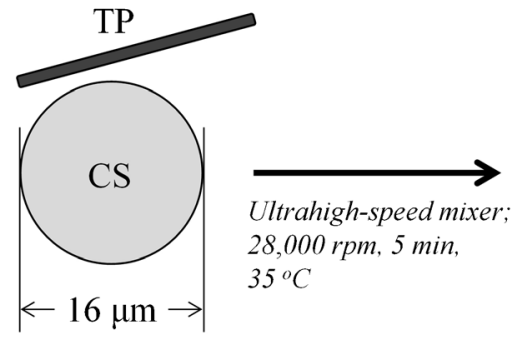

(Measured value; $15.9 \mu \mathrm{m}$ )

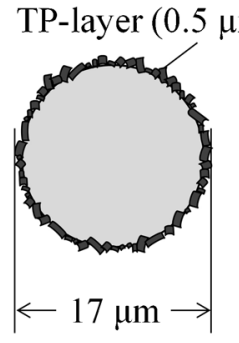

(Measured value; $16.2 \mu \mathrm{m}$ )

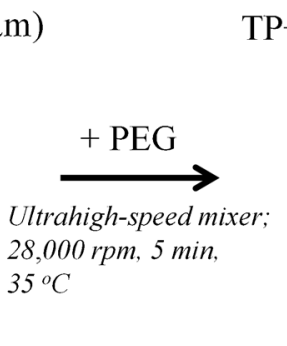

$\mathrm{TP}+\mathrm{PEG}$-layer $(1.5 \mu \mathrm{m})$

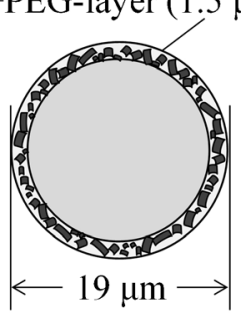

(Measured value; $17.1 \mu \mathrm{m}$ )

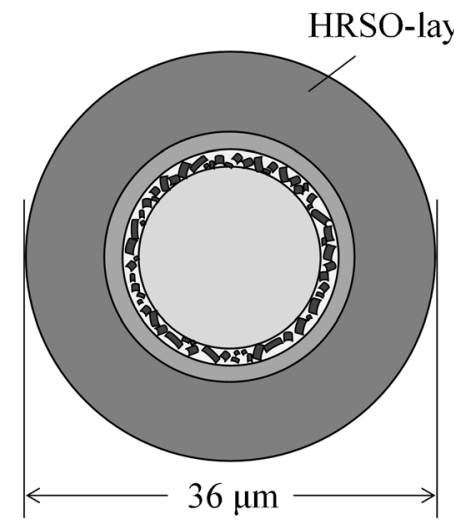

$\operatorname{er}(7.0 \mu \mathrm{m})$
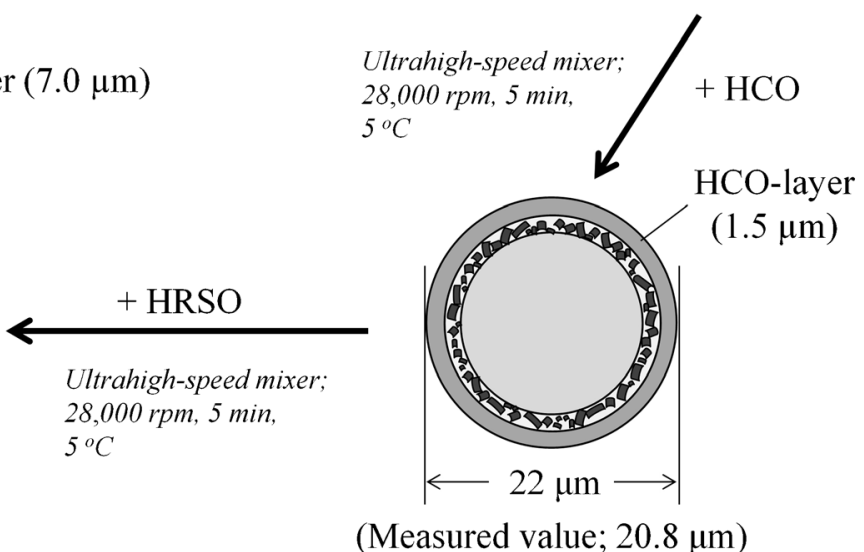

(Measured value; $32.4 \mu \mathrm{m}$ )

Fig. 12. Dry coating method for the preparation of controlled-release particles. The measured average particle diameter is indicated below each diagram

Since increasing the thickness of the HCO layer above $1.5 \mu \mathrm{m}$ was not possible, we attempted to seal the exterior of the $1.5-\mu \mathrm{m}$ HCO-coated particles using a hydrophobic layer of HRSO.

\section{Multi-layer Coating of the Controlled-Release Layer}

Because HRSO has a lower melting point than $\mathrm{HCO}$, and melted HRSO has a lower viscosity than melted HCO, we hoped that HRSO would seal the pores in the HCO layer. Figure 9 shows the shape and surface condition of particles

Table IV. The Average Particle Diameter and Geometric Standard Deviation

\begin{tabular}{llll}
\hline Particle & $\begin{array}{l}\text { Theoretical } \\
\text { diameter }(\mu \mathrm{m})\end{array}$ & $\begin{array}{l}\text { Average particle } \\
\text { diameter }(\mu \mathrm{m})\end{array}$ & $\begin{array}{l}\text { Geometric } \\
\text { standard } \\
\text { deviation }(-)\end{array}$ \\
\hline CS & - & $15.90 \pm 0.08$ & $0.14 \pm 0.00$ \\
CS-R & 19 & $17.05 \pm 0.19$ & $0.18 \pm 0.03$ \\
+HCO 1.5 $\mu \mathrm{m}$ & 22 & $20.76 \pm 1.16$ & $0.16 \pm 0.02$ \\
+HRSO 3 $\mu \mathrm{m}$ & 28 & $24.17 \pm 1.29$ & $0.17 \pm 0.02$ \\
+HRSO 5 $\mu \mathrm{m}$ & 32 & $26.88 \pm 0.15$ & $0.18 \pm 0.04$ \\
+HRSO 7 $\mu \mathrm{m}$ & 36 & $32.38 \pm 0.96$ & $0.25 \pm 0.02$ \\
\hline
\end{tabular}

Data represent the mean \pm standard deviation $(n=3)$

$C S$ cornstarch, $C S-R$ CS particles coated with a drug reservoir layer, $H C O$ hydrogenated castor oil, HRSO hydrogenated rapeseed oil obtained by mixing $1.5 \mu \mathrm{m}$ of HCO-coated particles with HRSO to create a 3.0-7.0- $\mu \mathrm{m}$ layer. Figure 9a shows $1.5-\mu \mathrm{m}$ HCO-coated particles, and Fig. 9b shows particles prepared by addition of $3.0 \mu \mathrm{m}$ of HRSO onto the HCO layer. The visual difference in surface conditions confirmed the formation of single-core particles with an external HRSO layer. Increasing the thickness of the HRSO layer to 5.0 and $7.0 \mu \mathrm{m}$ resulted in larger particle diameters, without causing granulation (Fig. 9c, d). We also observed fewer surface depressions and an overall smoothing of surface features when thicker coatings of HRSO were applied. The addition of any amount of HRSO appeared to laminate the particle surface, covering surface cracks and depressions. These results demonstrate that, in contrast to HCO, the addition of more HRSO is unlikely to cause granulation.

Figure 10 shows the DSC curves for HCO and HRSO. While the DSC curve for HRSO was one sharp peak, the curve for HCO showed a broad peak. According to previous reports $(17,18)$, the melting point of $\mathrm{HCO}$ was about $85^{\circ} \mathrm{C}$. Therefore, it was considered that the peak on the high-temperature side of the HCO DSC curve was due to pure $\mathrm{HCO}$, whereas the peak on the low-temperature side was due to HCO contaminants (polymorphism or analogous substances of $\mathrm{HCO}$ ). The melting point derived from the peak of the low-temperature side $\left(65.5^{\circ} \mathrm{C}\right)$ was slightly lower than that of $\mathrm{HRSO}\left(68.4^{\circ} \mathrm{C}\right)$. Upon further analysis of these DSC curves, we found that the enthalpy of fusion of $\mathrm{HCO}$ was $93.2 \mathrm{~J} / \mathrm{g}$, while that of $\mathrm{HRSO}$ was $154.4 \mathrm{~J} / \mathrm{g}$. This implied that $\mathrm{HCO}$ was easier to melt than HRSO when the same magnitude of energy, generated by the 
collisions and friction during mixing, was applied to the particle. For these reasons, it was considered that $\mathrm{HCO}$ would produce large quantities of molten liquid during low-temperature mixing more readily than HRSO. Conversely, the amount of molten HRSO that acted as a binding agent was small, as compared to HCO. Therefore, it was inferred that granulation does not occur with a $7.0-\mu \mathrm{m}$ coating of HRSO.

Figure 11 shows the dissolution of TP from particles coated with the indicated thickness of HRSO. The TP dissolution rate decreased as the thickness of the HRSO coating increased. For instance, the dissolution of TP from $7.0-\mu \mathrm{m}$ HRSO-coated particles was less than $10 \%$ after an 8 -h dissolution period. The dissolution rates from particles with HRSO layers thicker than $7.0 \mu \mathrm{m}$ were roughly the same as those from particles coated in $7.0 \mu \mathrm{m}$ of HRSO. With a 7.0- $\mu \mathrm{m}$ layer of HRSO, almost all cracks in the HCO layer were covered. We believe that this explained why TP dissolution was almost abolished.

\section{Fickian Diffusion}

For a more detailed understanding of how the formation of the HCO and HRSO layers affect drug dissolution, the dissolution behavior of TP was analyzed using an empirical formula proposed by Korsmeyer and Peppas $(19,20)$.

$\frac{\mathrm{M}_{t}}{\mathrm{M}_{\infty}}=K t^{\mathrm{n}}$

Where $\mathrm{M}_{t}$ is the amount of drug dissolved within time $t$, $\mathbf{M}_{\infty}$ is the total amount of drug dissolved over an infinite length of time, and $K$ is a rate constant. Generally, if the index $\mathrm{n}$ is 0.5 , then the drug dissolution corresponds to the Fickian diffusion model. If $\mathrm{n}<0.5$, the drug dissolution is pseudo-Fickian; whereby, the drug partially diffuses into the matrix. If $n=1$, then the swelling and relaxation of the matrix is the rate-determining step, and drug dissolution exhibits a zero-order release. For $0.5<\mathrm{n}<1$, the drug dissolution is non-Fickian. Both Fickian diffusion and matrix relaxation are believed to contribute to drug dissolution. Table III shows parameters obtained from analyses using this formula for the range $\mathrm{M}_{\mathrm{t}} / \mathrm{M}_{\infty}<0.6$ on the dissolution behavior of TP, as shown in Figs. 8 and 11. For the particles coated with $\mathrm{HCO}$ only, $K$ decreased as the thickness of the layer increased, and the value of $n$ was close to 1 at all thicknesses. This indicates that TP showed a steady release rate, which was independent of time. When the particles were coated with a 1.5- $\mu \mathrm{m}$ HCO layer followed by HRSO, $K$ was lower than that observed in particles coated with $\mathrm{HCO}$ only. The value of $\mathrm{n}$ was close to 0.5 or less, suggesting that dissolution of TP from CS-Rs coated in HCO and HRSO followed the Fickian diffusion theory.

These results confirm that dry coating can be used successfully to prepare sustained-release fine particles that were less than $50 \mu \mathrm{m}$ in diameter; these consisted of CS core particles coated in a drug reservoir layer (composed of TP and PEG), as well as an outer controlled-release bilayer (composed of $\mathrm{HCO}$ and $\mathrm{HRSO}$ ).

\section{Preparation of Controlled-Release Fine Particles via the Dry Coating Process}

Figure 12 illustrates the dry coating process used in this study for the preparation of sustained-release fine particles. Mixing CS with TP in an ultrahigh-speed mixer micronized large-diameter TP particles to a submicron size. Addition of water-soluble PEG to the mixture led to the formation of a drug reservoir layer (approximately $16 \mu \mathrm{m}$ in diameter) on the CS particles, which were already layered with TP and PEG. The drug reservoir layer was coated with hydrophobic HCO and HRSO to prepare single-core particles. Table IV shows the average particle diameter and geometric standard deviation at each stage of the preparation process. The observed particle diameter was slightly smaller than the theoretical value calculated using the prescription amounts, which was considered to be due to wear caused by particle collision during mixing. Moreover, only small variations were observed in the average particle diameter and geometric standard deviation at each stage, suggesting that particle preparation by this method was reproducible. The TP in the CS-Rs retained its crystalline structure, and dissolution of TP from the final product was less than $10 \%$ after $8 \mathrm{~h}$.

\section{CONCLUSION}

In this study, a dry coating process that did not use water or organic solvents was utilized to successfully prepare multilayer, single-core particles that were less than $50 \mu \mathrm{m}$ in diameter and contained both drug reservoir and controlled-release layers. We expect that this method will prove extremely useful for preparing sustained-release fine particles for use in multiple-unit, controlled-release tablets, capsules, and orally disintegrating tablets.

\section{ACKNOWLEDGMENTS}

We thank NOF Corp. for the kind gift of polyethylene glycol 20,000 and we thank Freund Corp., for the kind gifts of hydrogenated castor oil and hydrogenated rapeseed oil.

\section{REFERENCES}

1. Miyagawa $\mathrm{Y}$, Sato H, Okabe T, Nishiyama T, Miyajima M, Sunada H. In vivo performance of wax matrix particles prepared by a twin-screw compounding extruder. Drug Dev Ind Pharm. 1999;25:429-35.

2. Roy P, Shahiwala A. Multiparticulate formulation approach to pulsatile drug delivery: current perspectives. J Control Release. 2009;134:74-80.

3. Shimono N, Ueda M, Nakamura Y. Design of controlled release system with multi-layers of powder. Chem Pharm Bull. 2002;50:1169-75.

4. Pearnchob N, Bodmeier R. Dry powder coating of pellets with micronized Eudragit RS for extended drug release. Pharm Res. 2003;20:1970-6.

5. Fujimoto S, Uemura T, Fukumori Y. A trial of dry process coating using a twin screw continuous kneader. Powder Sci Eng. 2006;38:57-62.

6. Kondo K, Ito N, Niwa T, Danjo K. Design of sustained release fine particles using two-step mechanical powder processing: particle shape modification of drug crystals and dry particle coating with polymer nanoparticle agglomerate. Int J Pharm. 2013;453:523-32. 
7. Naganuma T, Nishida A, Sakamoto T, Kabasawa K, Imai K, Yuasa $\mathrm{H}$. Preparation of dry-coated particles with controlledrelease property using a new ultra-high-speed mixer. J Pharm Sci Technol Japan. 2009;69:452-60.

8. Habib W, Khankari R, Hontz J. Fast-dissolve drug delivery systems. Crit Rev Ther Drug Carrier Syst. 2000;17:61-72.

9. Engelen L, de Wijk RA, van der Bilt A, Prinz JF, Janssen AM, Bosman F. Relating particles and texture perception. Physiol Behav. 2005;86:111-7.

10. Yamada Y, Yonezawa Y, Sunada H. Evaluation of rapidly disintegrating tablet in the oral cavity prepared by dry compression method -high drug content tablets. J Pharm Sci Technol Japan. 2008;68:445-51.

11. Parkash V, Maan S, Deepika, Yadav SK, Hemlata, Jogpal V. Fast disintegrating tablets: opportunity in drug delivery system. J Adv Pharm Technol Res. 2011;2:223-35.

12. Venkatesh GM, Stevens PJ, Lai J. Development of orally disintegrating tablets comprising controlled-release multiparticulate beads. Drug Dev Ind Pharm. 2012;38:1428-40.

13. Kimura S, Uchida S, Kanada K, Namiki N. Effect of granule properties on rough mouth feel and palatability of orally disintegrating tablets. Int J Pharm. 2015;484:156-62.
14. Jono K, Ichikawa H, Miyamoto M, Fukumori Y. A review of particulate design for pharmaceutical powders and their production by spouted bed coating. Powder Technol. 2000;113:269-77.

15. Andrade EN. The viscosity of liquids. Nature. 1930;125:309-10.

16. Ennis BJ. A microlevel-based characterization of granulation phenomena. Powder Technol. 1991;65:257-72.

17. Özyazıcı M, Gökçe EH, Ertan G. Release and diffusional modeling of metronidazole lipid matrices. Eur J Pharm Biopharm. 2006;63:331-9.

18. Kowalski J, Kalb O, Joshi YM, Serajuddin ATM. Application of melt granulation technology to enhance stability of a moisture sensitive immediate-release drug product. Int J Pharm. 2009;381:56-61.

19. Korsmeyer RW, Gurny R, Doelker EM, Buri P, Peppas NA. Mechanism of solute release from porous hydrophilic polymers. Int J Pharm. 1983;15:25-35.

20. Ritger PL, Peppas NA. A simple equation for description of solute release II. Fickian and anomalous release from swellable devices. J Control Release. 1987;5:37-42. 